\title{
Effects of Thermal Diffusion and Radiation on Unsteady MHD Free Convection Flow Past an Infinite Heated Vertical Plate in a Porous Medium
}

\author{
S. Sivaiah, ${ }^{1}$ K. Anitha, ${ }^{2}$ and S. Venkataramana ${ }^{2}$ \\ ${ }^{1}$ Department of Mathematics, GITAM University, Hyderabad Campus, Hyderabad, Andhra Pradesh 502 329, India \\ ${ }^{2}$ Department of Mathematics, Sri Venkateswara University, Tirupati, Andhra Pradesh 517 502, India
}

Correspondence should be addressed to S. Sivaiah, sreddy7@yahoo.co.in

Received 25 September 2012; Accepted 27 October 2012

Academic Editors: R. R. Burnette and P. Trens

Copyright $\odot 2012$ S. Sivaiah et al. This is an open access article distributed under the Creative Commons Attribution License, which permits unrestricted use, distribution, and reproduction in any medium, provided the original work is properly cited.

\begin{abstract}
We have explored the influence of thermal diffusion and radiation on unsteady magnetohydrodynamic free convection flow past an infinite heated vertical plate in a porous medium. The governing boundary layer equations are written into a dimensionless form by similarity transformations. The transformed nonlinear differential equations are solved numerically with finite element methods. Numerical calculations are carried out for different values of dimensionless parameters. The results are presented graphically for velocity, temperature, and concentration profiles and show that the flow field and other quantities of physical interest are significantly influenced by these parameters.
\end{abstract}

\section{Introduction}

Flow through porous medium past infinite vertical plate is common in nature and has many applications in engineering and science. A number of workers have investigated such flows and excellent literature on the properties and phenomenon may be found in the literature [1-4]. For example, Soundalgekar [3] investigated the effects of free convection currents on the oscillatory type boundary layer flow past an infinite vertical plate with constant suction where the plate temperature differs from the free steam temperature.

Recently, attention has been on the effects of transversely applied magnetic field and thermal perturbation on the flow of electrically conducting viscous fluids such as plasma. Various properties associated with the interplay of magnetic fields and thermal perturbation in porous medium past vertical plate find useful applications in astrophysics, geophysical fluid dynamics, and engineering. Researches in these fields have been conducted by many investigators [5-9]. For example, Soundalgekar [10] investigated a two-dimensional steady free convection flow of an incompressible, viscous, and electrically conducting fluid past an infinite vertical porous plate with constant suction and plate temperature when the difference between the plate temperature and free stream is moderately large to cause free convection currents. In another study Israel-Cookey and Sigalo [11] investigated the problem of unsteady MHD past a semi-infinite vertical plate in an optically thin environment with simultaneous effects of radiation, free convection parameters, and timedependent suction. Chamka [12] investigated the unsteady convective heat and mass transfer past an infinite permeable moving plate with heat absorption where it was found that increase in Solutal Grashoff number enhanced the concentration buoyancy effects leading to an increase in the velocity. Anand Rao and Sivaiah [13] studied the chemical reaction effects on an unsteady MHD free convective flow past an infinite vertical porous plate with constant suction and heat source. Anand Rao et al. [14, 15] studied finite element solution of MHD transient flow past an impulsively started infinite horizontal porous plate in a rotating fluid with hall current and also studied heat and mass transfer in MHD flow of a viscous fluid past a vertical plate under oscillatory suction velocity.

The objective of the present chapter is to examine the effects of thermal diffusion and radiation on unsteady MHD free convection flow past an infinite heated vertical plate 
in a porous medium. The equations of continuity, linear momentum, energy, and diffusion, which govern the flow field, are solved by using Galerkin finite element method. Similarity solutions are then obtained numerically for various parameters, entering into the problem, and discussed from the physical point of view.

\section{Formulation of the Problem}

We consider the unsteady flow of an incompressible, viscous, and radiating hydromagnetic fluid past an infinite porous heated vertical plate with time-dependent suction in an optically thin environment. The physical model and the coordinate system are shown in Figure 1 . The $x^{\prime}$-axis is taken along the vertical infinite porous plate in the upward direction and the $y^{\prime}$-axis normal to the plate.

At time $t^{\prime}=0$, the plate is maintained at a temperature $T_{w}^{\prime}$, which is high enough to initiate radiative heat transfer. A constant magnetic field $H_{0}^{\prime 2}$ is maintained in the $y^{\prime}$ direction and the plate moves uniformly along the positive $x^{\prime}$ direction with velocity $U_{0}$. Under Boussinesq approximation the flow is governed by the following equations:

$$
\begin{gathered}
\frac{\partial w^{\prime}}{\partial y^{\prime}}=0 \\
\frac{\partial u^{\prime}}{\partial t^{\prime}}+w^{\prime} \frac{\partial u^{\prime}}{\partial y^{\prime}}=v \frac{\partial^{2} u^{\prime}}{\partial y^{\prime 2}}+\frac{\partial U^{\prime}}{\partial t^{\prime}} \\
-\left(\frac{\mu^{2} \sigma_{C} H_{0}^{\prime 2}}{\rho}+\frac{v}{K}\right)\left(u^{\prime}-U^{\prime}\right) \\
+g \beta\left(T^{\prime}-T_{\infty}^{\prime}\right)+g \beta^{*}\left(C^{\prime}-C_{\infty}^{\prime}\right), \\
\frac{\partial T^{\prime}}{\partial t^{\prime}}+w^{\prime} \frac{\partial T^{\prime}}{\partial y^{\prime}}=\frac{k}{\rho C_{P}}\left(\frac{\partial^{2} T^{\prime}}{\partial y^{\prime 2}}-\nabla q_{z^{\prime}}^{\prime}\right) \\
+\frac{\mu}{\rho C_{P}}\left(\frac{\partial u^{\prime}}{\partial y^{\prime}}\right)^{2}-\frac{Q_{o}}{\rho C_{P}}\left(T^{\prime}-T_{\infty}^{\prime}\right), \\
\frac{\partial^{2} q_{z^{\prime}}^{\prime}}{\partial y^{\prime 2}}-3 \alpha^{2} q_{z^{\prime}}^{\prime}-16 \alpha \sigma T_{\infty}^{3} \frac{\partial T^{\prime}}{\partial y^{\prime}}=0 \\
\frac{\partial C^{\prime}}{\partial t^{\prime}}+w^{\prime} \frac{\partial C^{\prime}}{\partial y^{\prime}}=D \frac{\partial^{2} C^{\prime}}{\partial y^{\prime 2}}+\frac{D_{m} k_{T}}{T_{m}} \frac{\partial^{2} T^{\prime}}{\partial y^{\prime 2}} .
\end{gathered}
$$

The boundary conditions are

$$
\begin{gathered}
u^{\prime}=0, \quad T^{\prime}=T_{w}^{\prime}, \quad C^{\prime}=C_{w}^{\prime} \quad \text { on } y^{\prime}=0, \\
u^{\prime}=0, \quad T^{\prime}=T_{\infty}^{\prime}, \quad C^{\prime}=C_{\infty}^{\prime} \quad \text { as } y^{\prime} \longrightarrow \infty,
\end{gathered}
$$

where $\left(u^{\prime}, v^{\prime}, w^{\prime}\right)$ are dimensional velocity components; $\left(x^{\prime}, y^{\prime}\right)$ is dimensional cartesian coordinates; $H_{0}^{\prime 2}$ is constant transverse magnetic field; $w_{0}^{\prime}$ is dimensional suction velocity; $T_{w}^{\prime}$ is wall temperature; $T_{\infty}^{\prime}$ is reference temperature; $U^{\prime}$ is dimensional free stream velocity; $t^{\prime}$ is dimensional time; $C_{w}^{\prime}$ is concentration near the plate; $C_{\infty}^{\prime}$ is concentration in the fluid for away from the plate the plate; $\rho$ is density; $g$ is acceleration due to gravity; $K$ is dimensional porosity parameter; $C_{p}$ is specific heat capacity; $k$ is thermal conductivity;

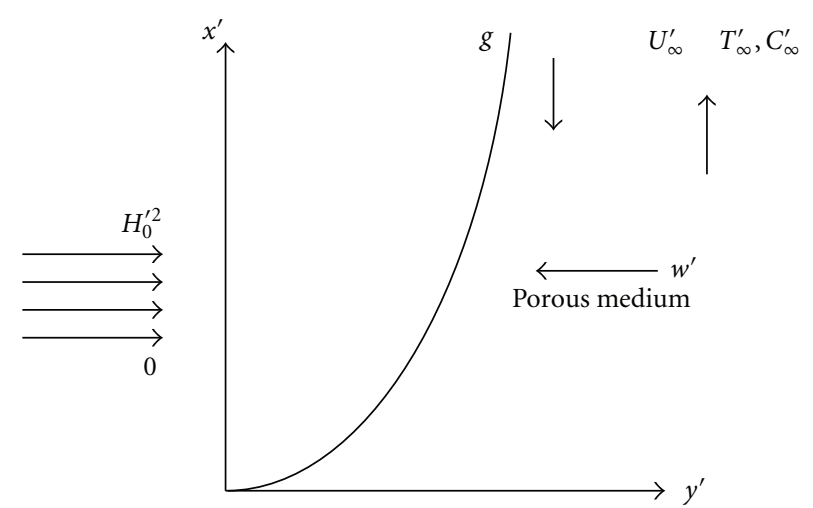

FIGURE 1: The physical model and coordinate system of the problem.

$q_{z}^{\prime}$ is radiative heat flux; $U_{0}$ is mean velocity of $U^{\prime}\left(t^{\prime}\right) ; \sigma_{C}$ is electrical conductivity; $Q_{0}$ is dimensional heat absorption coefficient; $v$ is kinematic coefficient; $\mu$ is permeability; $\varepsilon$ is time corrective parameter; $\beta$ is coefficients of volume expansion due to temperature; $\beta^{*}$ is coefficient of volume expansion due concentration; $D$ is chemical diffusivity; $D_{m}$ is molecular diffusivity; $k_{T}$ is mean absorption coefficient; $T_{m}$ is mean fluid temperature; $\alpha^{2}$ is absorption coefficient; $\omega^{\prime}$ is dimensional free stream frequency of oscillation.

Since the medium is optically thin with relatively low density and $\alpha \ll 1$, the radiative heat flux given by (4) in the spirit of Bestman [7] becomes

$$
\frac{\partial q_{z^{\prime}}^{\prime}}{\partial y^{\prime}}=4 \alpha^{2}\left(T^{\prime}-T_{\infty}^{\prime}\right)
$$

where

$$
\alpha^{2}=\int_{0}^{\infty} \delta \lambda \frac{\partial B}{\partial T^{\prime}} .
$$

Here $B$ is the Planck's constant, $\delta$ is the radiation absorption, and $\lambda$ is the frequency. Further, from (1) it is clear that the suction velocity $w^{\prime}$ at the plate is either a constant or a function of time only. So we assume it in the form

$$
w^{\prime}=-w_{0}^{\prime}\left(1+\varepsilon A e^{i \omega^{\prime} t^{\prime}}\right),
$$

where $A$ is small positive parameter and $\varepsilon$ is small such that $\varepsilon A \ll 1$, and the negative sign indicates that the suction velocity is towards the plate.

In view of (4), (8), and (9), (2), (3), and (5) become

$$
\begin{gathered}
\frac{1}{4} \frac{\partial u}{\partial t}-\left(1+\varepsilon A e^{i \omega t}\right) \frac{\partial u}{\partial y} \\
=\frac{1}{4} \frac{\partial U}{\partial t}+\frac{\partial^{2} u}{\partial y^{2}}-\left(M^{2}+\chi^{2}\right)(u-U)+\mathrm{Gr} \theta+\mathrm{GcC} \\
\frac{1}{4} \operatorname{Pr} \frac{\partial \theta}{\partial t}-\operatorname{Pr}\left(1+\varepsilon A e^{i \omega t}\right) \frac{\partial \theta}{\partial y} \\
=\left(\frac{\partial^{2}}{\partial y^{2}}-R^{2}\right) \theta+\operatorname{PrEc}\left(\frac{\partial u}{\partial y}\right)^{2}-\operatorname{Pr} S \theta \\
\frac{1}{4} \operatorname{Sc} \frac{\partial C}{\partial t}-\operatorname{Sc}\left(1+\varepsilon A e^{i \omega t}\right) \frac{\partial C}{\partial y}=\frac{\partial^{2} C}{\partial y^{2}}+(\operatorname{Sr})(\operatorname{Sc})\left(\frac{\partial^{2} \theta}{\partial y^{2}}\right)
\end{gathered}
$$


In order to write the governing equations and the boundary conditions in a dimensionless form, the following nondimensional quantities are introduced:

$$
\begin{gathered}
t=\frac{w_{0}^{\prime 2} t^{\prime}}{4 v}, \quad y=\frac{w_{0}^{\prime} y^{\prime}}{v}, \quad u=\frac{u^{\prime}}{U_{0}}, \\
\omega=\frac{4 v \omega^{\prime}}{w_{0}^{\prime 2}}, \quad U=\frac{U^{\prime}}{U_{0}}, \quad \theta=\frac{T^{\prime}-T_{\infty}^{\prime}}{T_{w}^{\prime}-T_{\infty}^{\prime}}, \\
\operatorname{Pr}=\frac{\mu c_{P}}{k}, \quad \mathrm{Gr}=\frac{g \beta v\left(T_{w}^{\prime}-T_{\infty}^{\prime}\right)}{U_{0} w_{0}^{\prime 2}}, \\
\mathrm{Gc}=\frac{g \beta^{*} v\left(C_{w}^{\prime}-C_{\infty}^{\prime}\right)}{U_{0} w_{0}^{\prime 2}}, \quad \mathrm{Ec}=\frac{U_{0}^{2}}{C_{P}\left(T_{w}^{\prime}-T_{\infty}^{\prime}\right)}, \\
R^{2}=\frac{4 \alpha^{2}}{\rho C_{P} k w_{0}^{\prime 2}}\left(T_{w}^{\prime}-T_{\infty}^{\prime}\right), \quad S=\frac{v \mathrm{Q}_{0}}{\rho c_{P} w_{0}^{\prime 2}}, \\
M^{2}=\frac{\mu^{2} \sigma_{C} H_{0}^{\prime 2}}{v \rho w_{0}^{\prime 2}}, \quad \\
\mathrm{Sr}=\frac{D_{m} k_{T}\left(T_{w}^{\prime}-T_{\infty}^{\prime}\right)}{v T_{m}\left(C_{w}^{\prime}-C_{\infty}^{\prime}\right)}, \quad \mathrm{Sc}=\frac{v}{D}, \\
C=\frac{\left(C^{\prime}-C_{\infty}^{\prime}\right)}{\left(C_{w}^{\prime}-C_{\infty}^{\prime}\right)}, \quad \chi^{2}=\frac{v^{2}}{K w_{0}^{\prime 2}},
\end{gathered}
$$

where $M^{2}$ is Hartmann number; $\operatorname{Pr}$ is Prandtl number; Sc is Schmidt number; Ec is Eckert number; $\theta$ is nondimensional temperature; $C$ is nondimensional species concentration; $\mathrm{Gr}$ is Grashof number; Gc is modified Grashof number; $\chi^{2}$ is Darcy number; $S r$ is Soret number; $R^{2}$ is radiation parameter; $S$ is heat source.

Equations (10), (11), and (12) are now subject to the boundary conditions

$$
\begin{gathered}
u=0, \quad \theta=1, \quad C=1 \quad \text { on } y=0, \\
u \longrightarrow 0, \quad \theta \longrightarrow 0, \quad C \longrightarrow 0 \quad \text { as } y \longrightarrow \infty .
\end{gathered}
$$

The mathematical statement of the problem is now complete and embodies the solution of (10), (11), and (12) subject to boundary conditions (14).

\section{Solution of the Problem}

By applying Galerkin finite element method for (10) over the element $(e)\left(y_{j} \leq y \leq y_{k}\right)$ is

$$
\begin{gathered}
\int_{y_{j}}^{y_{k}}\left\{N^{(e)^{T}}\left[4 \frac{\partial^{2} u^{(e)}}{\partial y^{2}}-\frac{\partial u^{(e)}}{\partial t}+4 B \frac{\partial u^{(e)}}{\partial y}-D u^{(e)}+P\right]\right\} d y \\
=0
\end{gathered}
$$

where $P=(\partial U / \partial t)+4(\mathrm{Gr}) \theta+4(\mathrm{Gc}) C+D U, B=1+\varepsilon A e^{i \omega t}$, and $D=4\left(M^{2}+\chi^{2}\right)$.
Integrating the first term in (15) by parts, one obtains

$$
\begin{aligned}
4 N^{(e)^{T}}\left\{\frac{\partial u^{(e)}}{\partial y}\right\}_{y_{j}}^{y_{k}} & \\
-\int_{y_{j}}^{y_{k}}\left\{4 \frac{\partial N^{(e)^{T}}}{\partial y} \frac{\partial u^{(e)}}{\partial y}\right. & \\
& \left.+N^{(e)^{T}}\left(\frac{\partial u^{(e)}}{\partial t}-4 B \frac{\partial u^{(e)}}{\partial y}+D u^{(e)}-P\right)\right\} d y=0 .
\end{aligned}
$$

Neglecting the first term in (16), one gets

$$
\begin{aligned}
\int_{y_{j}}^{y_{k}}\left\{4 \frac{\partial N^{(e)^{T}}}{\partial y} \frac{\partial u^{(e)}}{\partial y}\right. & \\
& \left.+N^{(e)^{T}}\left(\frac{\partial u^{(e)}}{\partial t}-4 B \frac{\partial u^{(e)}}{\partial y}+D u^{(e)}-P\right)\right\} d y=0 .
\end{aligned}
$$

Let $u^{(e)}=N^{(e)} \phi^{(e)}$ be the linear piecewise approximation solution over the element $(e)\left(y_{j} \leq y \leq y_{k}\right)$, where $N^{(e)}=$ $\left[N_{j} N_{k}\right], \phi^{(e)}=\left[u_{j} u_{k}\right]^{T}, N_{j}=\left(y_{k}-y\right) /\left(y_{k}-y_{j}\right)$, and $N_{k}=$ $\left(y-y_{j}\right) /\left(y_{k}-y_{j}\right)$ are the basis functions. One obtains

$$
\begin{aligned}
4 \int_{y_{j}}^{y_{k}}\left\{\left[\begin{array}{llll}
N_{j}^{\prime} & N_{j}^{\prime} & N_{j}^{\prime} & N_{k}^{\prime} \\
N_{j}^{\prime} & N_{k}^{\prime} & N_{k}^{\prime} & N_{k}^{\prime}
\end{array}\right]\left[\begin{array}{l}
u_{j} \\
u_{k}
\end{array}\right]\right\} d y \\
+\int_{y_{j}}^{y_{k}}\left\{\left[\begin{array}{llll}
N_{j} & N_{j} & N_{j} & N_{k} \\
N_{j} & N_{k} & N_{k} & N_{k}
\end{array}\right]\left[\begin{array}{l}
\dot{u}_{j} \\
\dot{u}_{k}
\end{array}\right]\right\} d y \\
\quad-4 B \int_{y_{j}}^{y_{k}}\left\{\left[\begin{array}{llll}
N_{j} & N_{j}^{\prime} & N_{j} & N_{k}^{\prime} \\
N_{j}^{\prime} & N_{k} & N_{k}^{\prime} & N_{k}
\end{array}\right]\left[\begin{array}{l}
u_{j} \\
u_{k}
\end{array}\right]\right\} d y \\
+D \int_{y_{j}}^{y_{k}}\left\{\left[\begin{array}{llll}
N_{j} & N_{j} & N_{j} & N_{k} \\
N_{j} & N_{k} & N_{k} & N_{k}
\end{array}\right]\left[\begin{array}{l}
u_{j} \\
u_{k}
\end{array}\right]\right\} d y \\
=P \int_{y_{j}}^{y_{k}}\left[\begin{array}{l}
N_{j} \\
N_{k}
\end{array}\right] d y .
\end{aligned}
$$

Simplifying, we get

$$
\begin{aligned}
\frac{4}{l(e)^{2}} & {\left[\begin{array}{cc}
1 & -1 \\
-1 & 1
\end{array}\right]\left[\begin{array}{l}
u_{j} \\
u_{k}
\end{array}\right]+\frac{1}{6}\left[\begin{array}{ll}
2 & 1 \\
1 & 2
\end{array}\right]\left[\begin{array}{l}
\dot{u}_{j} \\
\dot{u}_{k}
\end{array}\right] } \\
& -\frac{4 B}{2 l^{(e)}}\left[\begin{array}{ll}
-1 & 1 \\
-1 & 1
\end{array}\right]\left[\begin{array}{l}
u_{j} \\
u_{k}
\end{array}\right]+\frac{D}{6}\left[\begin{array}{ll}
2 & 1 \\
1 & 2
\end{array}\right]\left[\begin{array}{l}
u_{j} \\
u_{k}
\end{array}\right] \\
= & \frac{P}{2}\left[\begin{array}{l}
1 \\
1
\end{array}\right],
\end{aligned}
$$

where prime and dot denote differentiation with respect to $y$ and time $t$, respectively. Assembling the element equations 


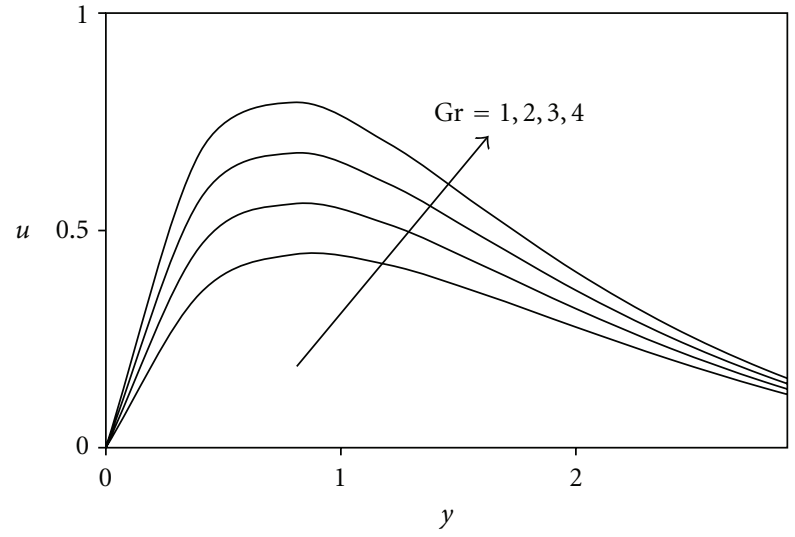

Figure 2: Velocity profiles for different values of Gr.

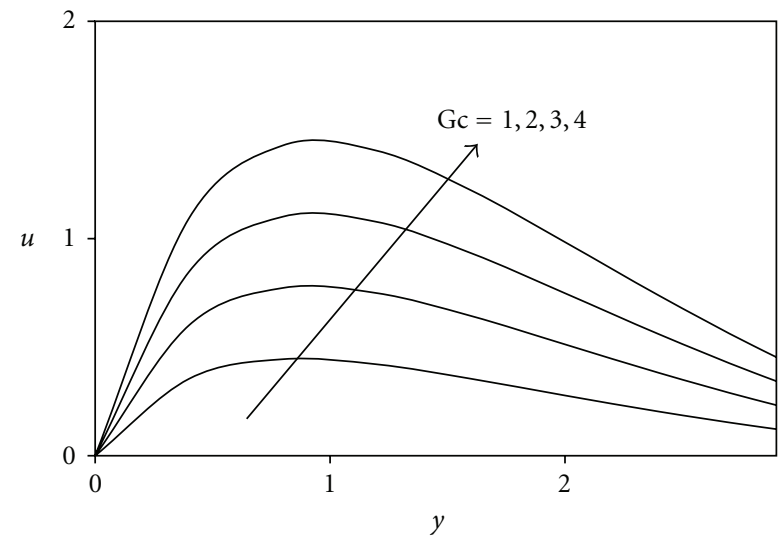

FIgURE 3: Velocity profiles for different values of Gc.

for two consecutive elements $\left(y_{i-1} \leq y \leq y_{i}\right)$ and $\left(y_{i} \leq y \leq\right.$ $\left.y_{i+1}\right)$, one obtains

$$
\begin{aligned}
& \frac{4}{l(e)^{2}}\left[\begin{array}{ccc}
1 & -1 & 0 \\
-1 & 2 & -1 \\
0 & -1 & 1
\end{array}\right]\left[\begin{array}{c}
u_{i-1} \\
u_{i} \\
u_{i+1}
\end{array}\right] \\
& +\frac{1}{6}\left[\begin{array}{ccc}
2 & 1 & 0 \\
1 & 4 & 1 \\
0 & 1 & 2
\end{array}\right]\left[\begin{array}{c}
\dot{u}_{i-1} \\
\dot{u}_{i} \\
\dot{u}_{i+1}
\end{array}\right] \\
& -\frac{4 B}{2 l^{(e)}}\left[\begin{array}{ccc}
-1 & 1 & 0 \\
-1 & 0 & 1 \\
0 & -1 & 1
\end{array}\right]\left[\begin{array}{c}
u_{i-1} \\
u_{i} \\
u_{i+1}
\end{array}\right] \\
& +\frac{D}{6}\left[\begin{array}{lll}
2 & 1 & 0 \\
1 & 4 & 1 \\
0 & 1 & 2
\end{array}\right]\left[\begin{array}{c}
u_{i-1} \\
u_{i} \\
u_{i+1}
\end{array}\right]=\frac{P}{2}\left[\begin{array}{l}
1 \\
2 \\
1
\end{array}\right] .
\end{aligned}
$$

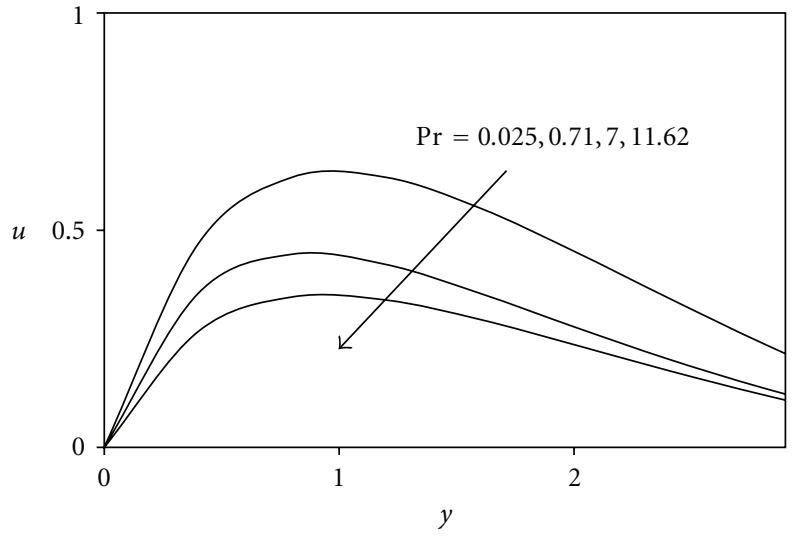

FIGURE 4: Velocity profiles for different values of Pr.

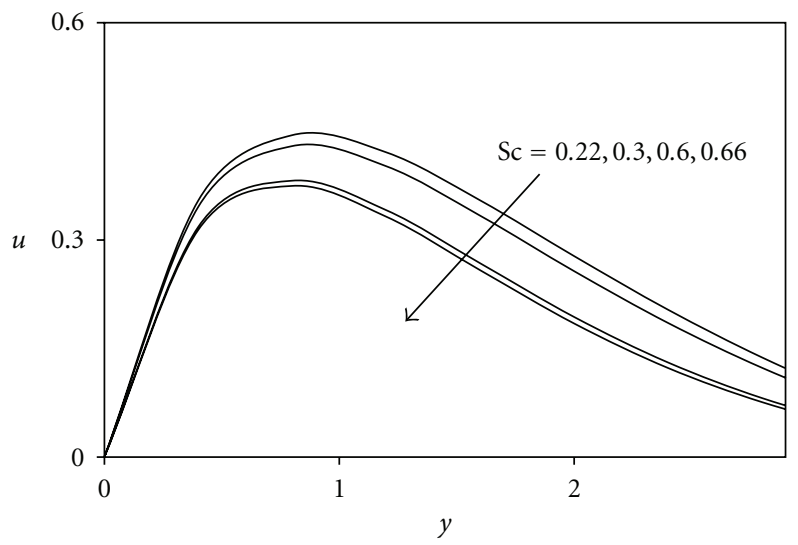

FIGURE 5: Velocity profiles for different values of Sc.

Now put row corresponding to the node $i$ to zero, from (20) the difference schemes with $l^{(e)}=h$ are

$$
\begin{aligned}
\frac{4}{h^{2}}[- & \left.u_{i-1}+2 u_{i}-u_{i+1}\right] \\
& +\frac{1}{6}\left[\dot{u}_{i-1}+4 \dot{u}_{i}+\dot{u}_{i+1}\right] \\
& -\frac{4 B}{2 h}\left[-u_{i-1}+u_{i+1}\right] \\
& +\frac{D}{6}\left[u_{i-1}+4 u_{i}+u_{i+1}\right]=P .
\end{aligned}
$$

Applying the trapezoidal rule, the following system of equations in Crank-Nicholson method is obtained

$$
\begin{aligned}
& A_{1} u_{i-1}^{n+1}+A_{2} u_{i}^{n+1}+A_{3} u_{i+1}^{n+1} \\
& \quad=A_{4} u_{i-1}^{n}+A_{5} u_{i}^{n}+A_{6} u_{i+1}^{n}+P^{*} .
\end{aligned}
$$




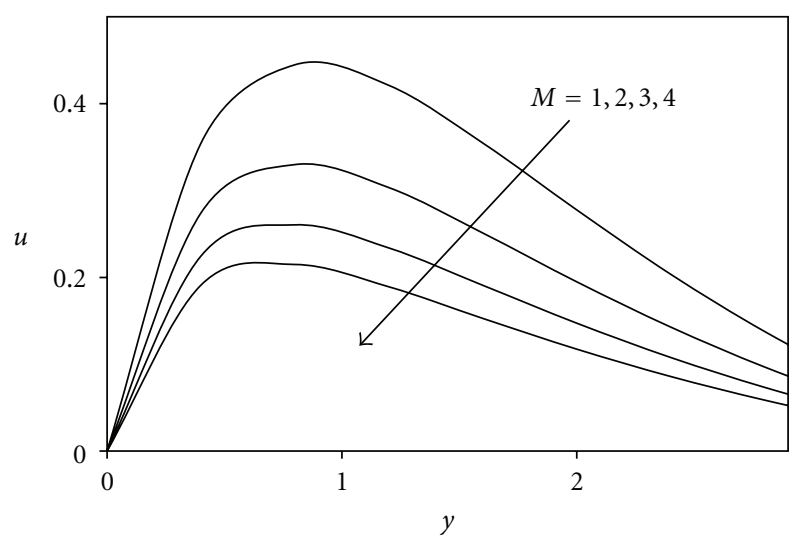

Figure 6: Velocity profiles for different values of $M$.

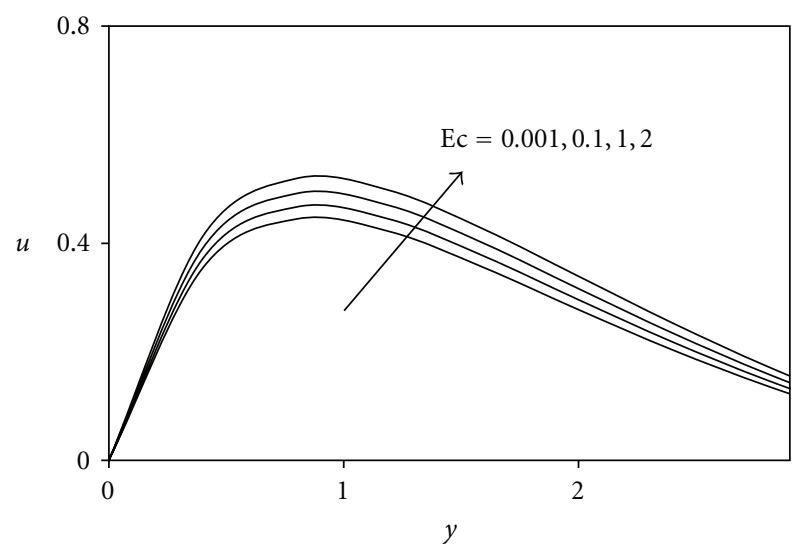

Figure 7: Velocity profiles for different values of Ec.

Now from (11) and (12) the following equations are obtained:

$$
\begin{aligned}
& B_{1} \theta_{i-1}^{n+1}+B_{2} \theta_{i}^{n+1}+B_{3} \theta_{i+1}^{n+1} \\
&= B_{4} \theta_{i-1}^{n}+B_{5} \theta_{i}^{n}+B_{6} \theta_{i+1}^{n}+Q^{*}, \\
& C_{1} C_{i-1}^{n+1}+C_{2} C_{i}^{n+1}+C_{3} C_{i+1}^{n+1} \\
&=C_{4} C_{i-1}^{n}+C_{5} C_{i}^{n}+C_{6} C_{i+1}^{n}+R^{* *},
\end{aligned}
$$

where

$$
\begin{gathered}
A_{1}=2+12 B r h+D k-24 r, \\
A_{2}=8+4 D k+48 r, \\
A_{3}=2-12 B r h+D k-24 r, \\
A_{4}=2-12 B r h-D k+24 r, \\
A_{5}=8-4 D k-48 r, \\
A_{6}=2+12 B r h+D k+24 r, \\
B_{1}=2(\operatorname{Pr})+12(\operatorname{Pr}) B r h+4 R^{2} k-24 r+4 S(\operatorname{Pr}) k, \\
B_{2}=8(\operatorname{Pr})+48 r+16 R^{2} k+16 S(\operatorname{Pr}) k,
\end{gathered}
$$

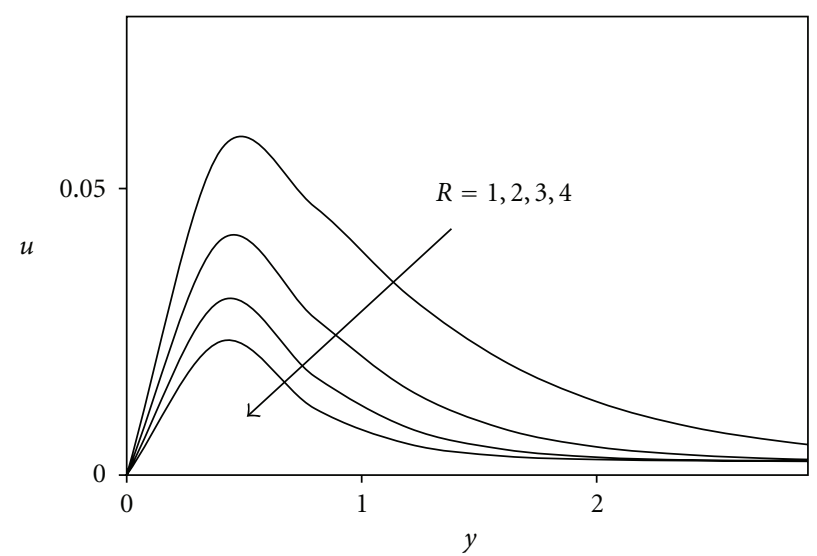

FIGURE 8: Velocity profiles for different values of $R$.

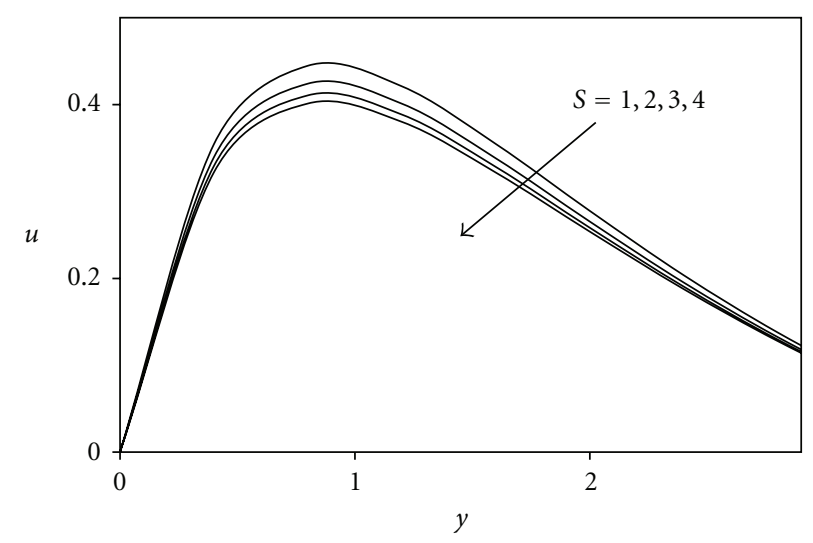

FIgURE 9: Velocity profiles for different values of $S$.

$$
\begin{gathered}
B_{3}=2(\operatorname{Pr})-12(\operatorname{Pr}) B r h+4 R^{2} k-24 r+4 S(\operatorname{Pr}) k, \\
B_{4}=2(\operatorname{Pr})-12(\operatorname{Pr}) B r h-4 R^{2} k+24 r-4 S(\operatorname{Pr}) k, \\
B_{5}=8(\operatorname{Pr})-48 r-16 R^{2} k-16 S(\operatorname{Pr}) k, \\
B_{6}=2(\operatorname{Pr})+12(\operatorname{Pr}) B r h-4 R^{2} k+24 r-4 S(\operatorname{Pr}) k, \\
C_{1}=2(\mathrm{Sc})+12(\operatorname{Pr}) B r h-24 r, \\
C_{2}=8(\mathrm{Sc})+48 r, \\
C_{3}=2(\mathrm{Sc})-12(\mathrm{Sc}) B r h-24 r, \\
C_{4}=2(\mathrm{Sc})-12(\mathrm{Sc}) B r h+24 r, \\
C_{5}=8(\mathrm{Sc})-48 r, \\
C_{6}=2(\mathrm{Sc})+12(\mathrm{Sc}) B r h+24 r, \\
P^{*}=12 P k=12 k((\partial U / \partial t)+4(\mathrm{Gr}) \theta+4(\mathrm{Gc}) C+D U), \\
Q^{*}=12 Q k=48(\mathrm{Pr}) k(\mathrm{Ec})(\partial u / \partial y)^{2}, \\
R^{* *}=12 R^{*} k=48(\mathrm{Sc})(\mathrm{Sr}) k\left(\partial^{2} \theta / \partial y^{2}\right) .
\end{gathered}
$$

Here $r=k / h^{2}$ and $h, k$ are mesh sizes along $y$-direction and time-direction, respectively. Index $i$ refers to space and $j$ refers to the time. In (22) and (23), taking $i=1(1) n$ and 


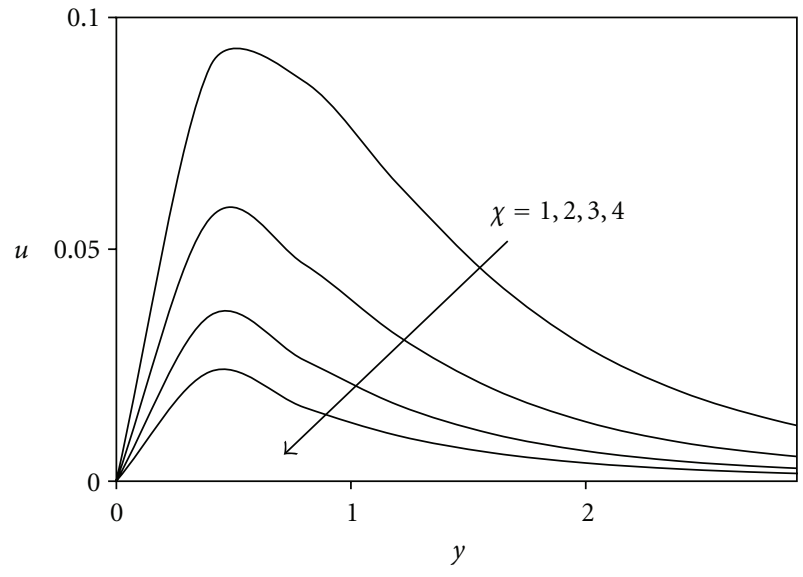

Figure 10: Velocity profiles for different values of $\chi$.

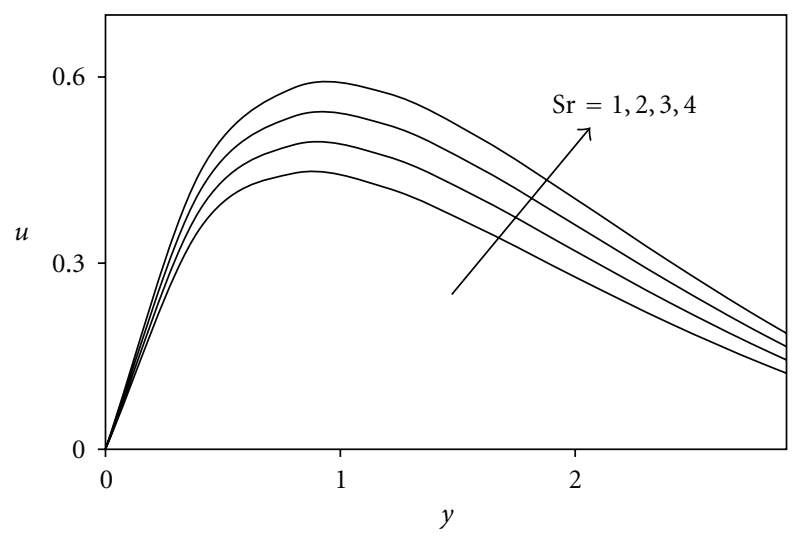

FIgURE 11: Velocity profiles for different values of Sr.

using boundary conditions (14), then the following system of equations is obtained

$$
A_{i} X_{i}=B_{i} \quad i=1(1) 3
$$

where $A_{i}^{\prime}$ s are matrices of order $n$ and $X_{i}, B_{i}^{\prime}$ s are column matrices having $n$-components. The solutions of the above system of equations are obtained by using Thomas algorithm for velocity, temperature, and concentration. Also, numerical solutions for these equations are obtained by $C$-programme. In order to prove the convergence and stability of Galerkin finite element method, the same $C$-programme was run with smaller values of $h$ and $k$; no significant change was observed in the values of $u, \theta$, and $C$. Hence the Galerkin finite element method is stable and convergent.

\section{Results and Discussion}

In the previous sections, we have formulated and solved the problem of an unsteady MHD free convection flow past an infinite heated vertical plate in a porous medium with radiation. By invoking, the optically thin differential approximation for the radiative heat flux in the energy equation. In the numerical computation, the Prandtl number $(\operatorname{Pr}=0.71)$ which corresponds to air and various values of the material

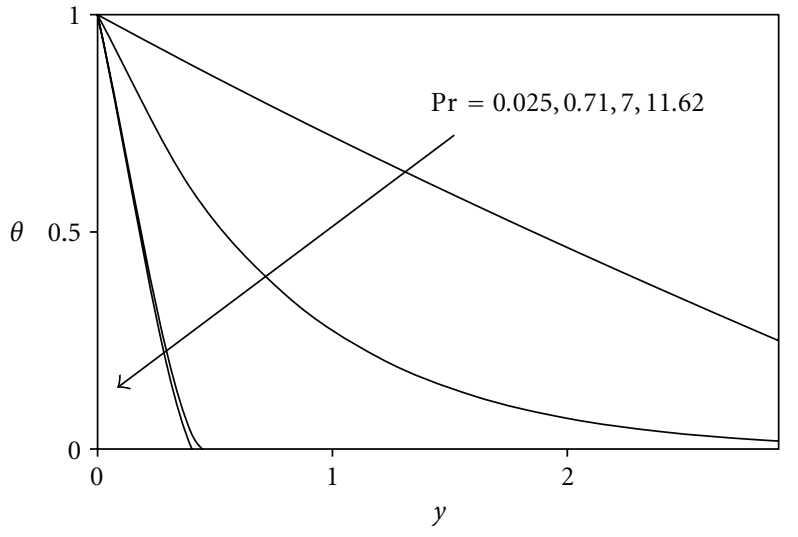

Figure 12: Temperature profiles for different values of Pr.

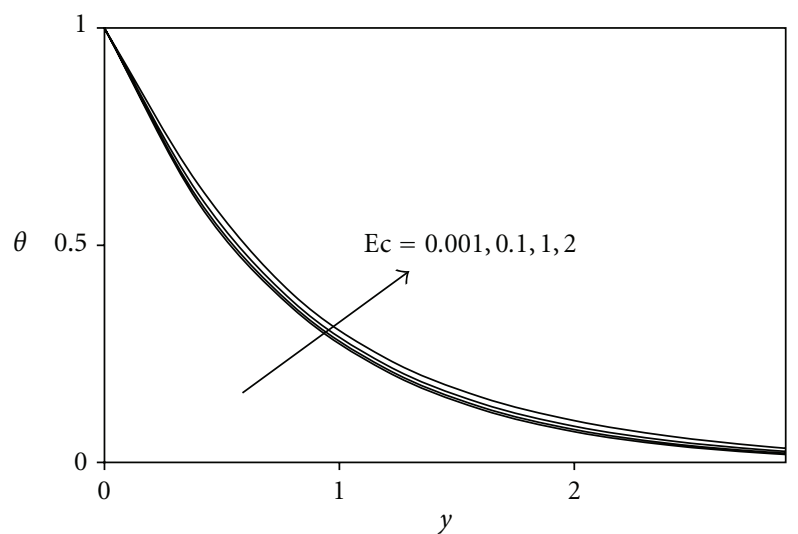

Figure 13: Temperature profiles for different values of Ec.

parameters are used. In addition, the boundary condition $y \rightarrow \infty$ is approximated by $y_{\max }=2$, which is sufficiently large for the velocity to approach the relevant stream velocity. The temperature and the species concentration are coupled to the velocity via Grashof number $(\mathrm{Gr})$ and modified Grashof number (Gc) as seen in (9). For various values of Grashof number and modified Grashof number, the velocity profiles $u$ are plotted in Figures 2 and 3. The Grashof number (Gr) signifies the relative effect of the thermal buoyancy force to the viscous hydrodynamic force in the boundary layer. As expected, it is observed that there is a rise in the velocity due to the enhancement of thermal buoyancy force. Also, as $(\mathrm{Gr})$ increases, the peak value of the velocity increases rapidly near the porous plate and then decays smoothly to the free stream velocity. The modified Grashof number (Gc) defines the ratio of the species buoyancy force to the viscous hydrodynamic force. As expected, the fluid velocity increases and the peak value is more distinctive due to the increase in the species buoyancy force. The velocity distribution attains a distinctive maximum value in the vicinity of the plate and then decreases properly to approach the free stream value. It is noticed that the velocity increases with the increasing values of modified Grashof number (Gc). Figure 4 illustrates the velocity profiles for different values of Prandtl number Pr. The numerical results show that the effect of increasing 


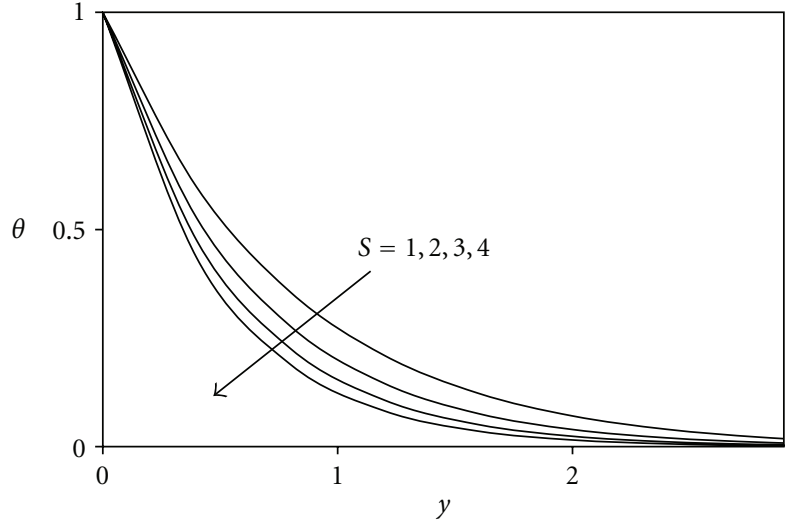

Figure 14: Temperature profiles for different values of $S$.

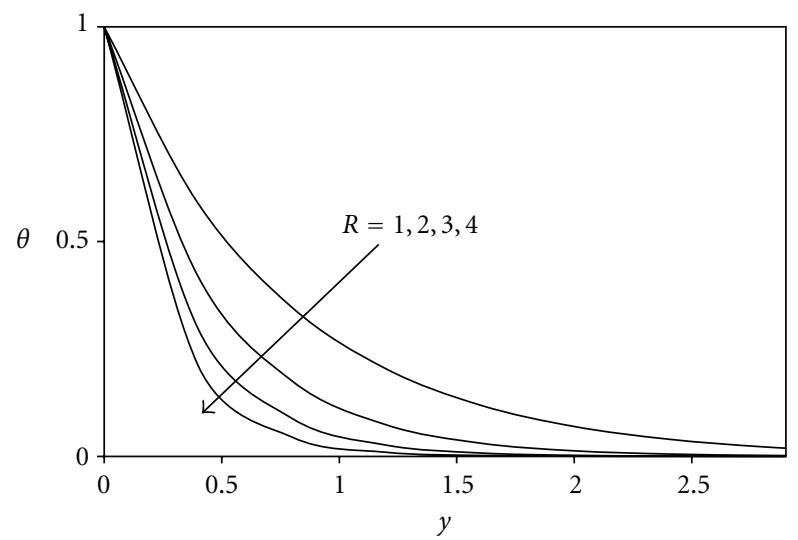

FIGURE 15: Temperature profiles for different values of $R$.

values of Prandtl number results in decreasing velocity. The nature of velocity profiles in the presence of foreign species such as hydrogen $(\mathrm{Sc}=0.22)$, helium $(\mathrm{Sc}=0.30)$, water vapour $(\mathrm{Sc}=0.60)$, and oxygen $(\mathrm{Sc}=0.66)$ is shown in Figure 5 . The flow field suffers a decrease in primary velocity at all points in the presence of heavier diffusing species. The effect of the magnetic field parameter $M$ is shown in Figure 6 in case of cooling of the plate. It is observed that the velocity of the fluid decreases with the increase of the magnetic field parameter values. The decrease in the velocity as the Hartmann number $M$ increases is because the presence of a magnetic field in an electrically conducting fluid introduces a force called the Lorentz force, which acts against the flow if the magnetic field is applied in the normal direction, as in the present study. This resistive force slows down the fluid velocity component as shown in Figure 6 . The influence of the viscous dissipation parameter, that is, the Eckert number Ec on the velocity, and temperature are shown in Figures 7 and 13, respectively. The Eckert number Ec expresses the relationship between the kinetic energy in the flow and the enthalpy. It embodies the conversion of kinetic energy into internal energy by work done against the viscous fluid stresses. Greater viscous dissipative heat causes a rise in the temperature as well as the velocity.

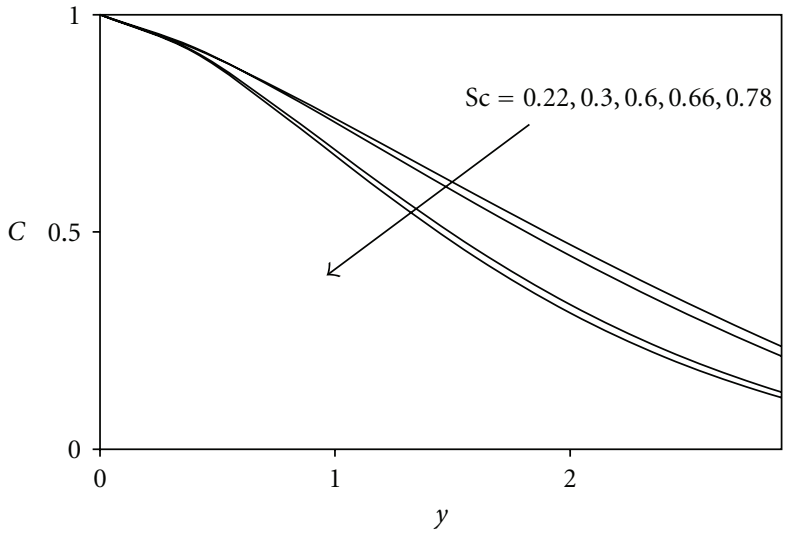

Figure 16: Concentration profiles for different values of Sc.

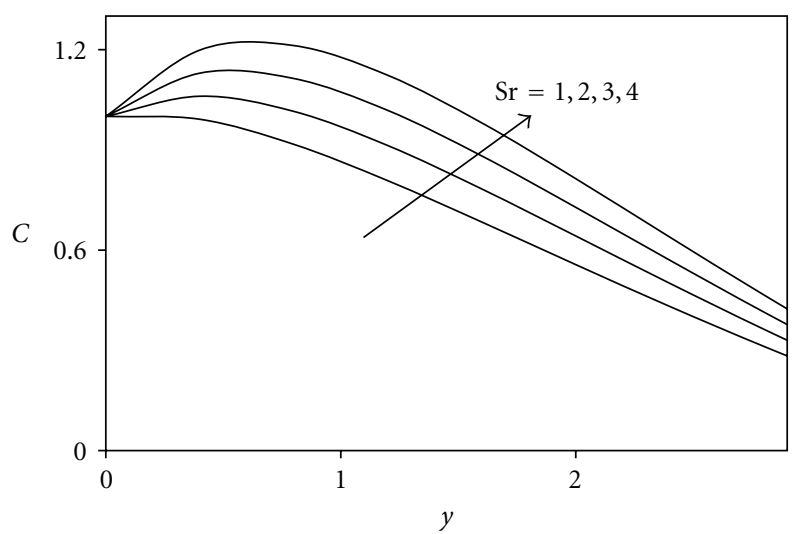

FIGURE 17: Concentration profiles for different values of Sr.

The effect of the thermal radiation parameter $R$ on the primary velocity and temperature profiles in the boundary layer is illustrated in Figures 8 and 15, respectively. Increasing the thermal radiation parameter $R$ produces significant increase in the thermal condition of the fluid and its thermal boundary layer. This increase in the fluid temperature induces more flow in the boundary layer causing the velocity of the fluid there to increase. Figures 9 and 14 have been plotted to depict the variation of velocity and temperature profiles against $y$ for different values of heat source parameter $S$ by fixing other physical parameters. From this graph we observe that velocity and temperature decrease with increase in the heat source parameter $S$ because when heat is absorbed, the buoyancy force decreases the temperature profiles. Figure 10 shows the effects of Darcy number $\chi$ on the velocity profiles for cooling as well as heating the plate. For a cooling plate, fluid velocity increases, whereas for a heating plate it decreases with increase of $\chi$. Darcy number is the measurement of the porosity of the medium. As the porosity of the medium increases, the value of $\chi$ increases. For large porosity of the medium, fluid gets more space to flow, as a consequence its velocity increases. The variations of tangential velocity distribution with $y$ for different values of the Soret number $(\mathrm{Sr})$ are shown in Figure 11. It can be clearly seen that the velocity distribution in the boundary 
layer increases with the Soret number. Figure 12 illustrates the temperature profiles for different values of Prandtl number Pr. It is observed that the temperature decreases as the Prandtl number increases. The reason is that smaller values of $\operatorname{Pr}$ are equivalent to increase in the thermal conductivity of the fluid and therefore heat is able to diffuse away from the heated surface more rapidly for higher values of Pr. Hence in the case of smaller Prandtl number the thermal boundary layer is thicker and the rate of heat transfer is reduced. The effects of Schmidt number (Sc) and Soret number $(\mathrm{Sr})$ on the concentration field are presented in Figures 16 and 17. Figure 16 shows that the concentration field is due to variation in Schmidt number (Sc) for the gasses hydrogen, helium, water vapour, oxygen, and ammonia. It is observed that the concentration field is steady for hydrogen and falls rapidly for oxygen and ammonia in comparison to water vapour. Thus hydrogen can be used for maintaining effective concentration field and water vapour can be used for maintaining normal concentration field. In Figure 17, it is observed that an increase in the Soret number ( $\mathrm{Sr}$ ) leads to increase in the concentration field.

\section{Conclusions}

In conclusion, therefore, the flow of an unsteady MHD free convection past an infinite heated vertical plate in a porous medium under the simultaneous effects of thermal diffusion and radiation is affected by the material parameters. The governing equations are approximated to a system of linear partial differential equations by using Galerkin finite element method. The results are presented graphically and we can conclude that the flow field and the quantities of physical interest are significantly influenced by these parameters.

(1) The velocity increases as Grashof number Gr, modified Grashof number Gc, Eckert number, thermal radiation parameter $R$, Darcy parameter $\chi$, Ec, and Soret number $\mathrm{Sr}$ increase. However, the velocity was found to be decreasing as the Hartmann number $M$, Prandtl number Pr, Schmidt number Sc, and heat source parameter $S$ are increasing.

(2) The fluid temperature was found to be decreasing as the heat source parameter $S$ and Prandtl number Pr are increasing and found to be increasing as Eckert number Ec, and thermal radiation parameter $R$ are increasing.

(3) The fluid concentration was found to be decreasing as the Schmidt number Sc and increasing as the Soret number $\mathrm{Sr}$ is increasing.

\section{References}

[1] M. J. Lightill, "The response of laminar skin-friction and heat transfer fluctuations in the stream velocity," Proceeding of the Royal Socity London A, vol. 224, pp. 1-23, 1954.

[2] I. Pop and V. M. Soundalgekar, "On unsteady boundary layers in a rotating flow," IMA Journal of Applied Mathematics, vol. 15, no. 3, pp. 343-349, 1975.

[3] V. M. Soundalgekar, "Free-convection effects on the oscillatory flow past an infinite vertical porous plate with constant suction," Proceeding of the Royal Socity London A, vol. 333, pp. 25-36, 1973.

[4] H. S. Takhar, R. S. R. Gorla, and V. M. Soundalgekar, "Radiation effects on MHD free convection flow of a gas past a semi-infinite vertical plate," International Journal of Numerical Methods for Heat and Fluid Flow, vol. 6, no. 2, pp. 77-83, 1996.

[5] K. D. Alagoa, G. Tay, and T. M. Abbey, "Radiative and freeconvective effects of a MHD flow through a porous medium between infinite parallel plates with time-dependent suction," Astrophysics and Space Science, vol. 260, no. 4, pp. 455-468, 1998.

[6] A. R. Bestman and S. K. Adjepong, "Unsteady hydromagnetic free-convection flow with radiative heat transfer in a rotating fluid-I. Incompressible optically thin fluid," Astrophysics and Space Science, vol. 143, no. 1, pp. 73-80, 1988.

[7] A. R. Bestman, "Transient effect on laminar convection to a radiating gas past a vertical flat plate," Modelling, Simulation \& Control B, vol. 3, no. 2, pp. 1-10, 1985.

[8] A. C. L. Cogley, W. G. Vincenti, and E. S. Gillies, "Differential approximation for radiative heat transfer in a non-linear equations-grey gas near equilibrium," Journal of American Institue Aeronuat and Astronaut, vol. 6, pp. 551-553, 1968.

[9] Y. J. Kim, "Unsteady MHD convective heat transfer past a semi-infinite vertical porous moving plate with variable suction," International Journal of Engineering Science, vol. 38, no. 8, pp. 833-845, 2000.

[10] V. M. Soundalgekar, "Free-convection effects on steady MHD flow past a vertical porous plate," Journal of Fluid Mechanics, vol. 66, no. 3, pp. 541-551, 1974.

[11] C. Israel-Cookey and F. B. Sigalo, "On the unsteady MHD freeconvection flow past semi-infinite heated porous vertical plate with time-dependant suction and radiative heat transfer," Modelling, Measurement \& Control B, vol. 72, no. 1-2, pp. 5165, 2003.

[12] A. J. Chamkha, "Unsteady MHD convective heat and mass transfer past a semi-infinite vertical permeable moving plate with heat absorption," International Journal of Engineering Science, vol. 42, no. 2, pp. 217-230, 2004.

[13] J. Anand Rao and S. Shivaiah, "Chemical reaction effects on an unsteady MHD free convective flow past an infinite vertical porous plate with constant suction and heat source," International Journal of Applied Mathematics and Mechanics, vol. 7, no. 8, pp. 98-118, 2011.

[14] J. Anand Rao, R. Srinivasa Raju, and S. Sivaiah, "Finite element solution of MHD transient flow past an impulsively started infinite horizontal porous plate in a rotating fluid with hall current," Journal of Applied Fluid Mechanics, vol. 5, no. 3, pp. 105-112, 2012.

[15] J. Anand Rao, R. Srinivasa Raju, and S. Sivaiah, "Finite element solution of heat and mass transfer in MHD flow of a viscous fluid past a vertical plate under oscillatory suction velocity," Journal of Applied Fluid Mechanics, vol. 5, no. 3, pp. 1-10, 2012. 

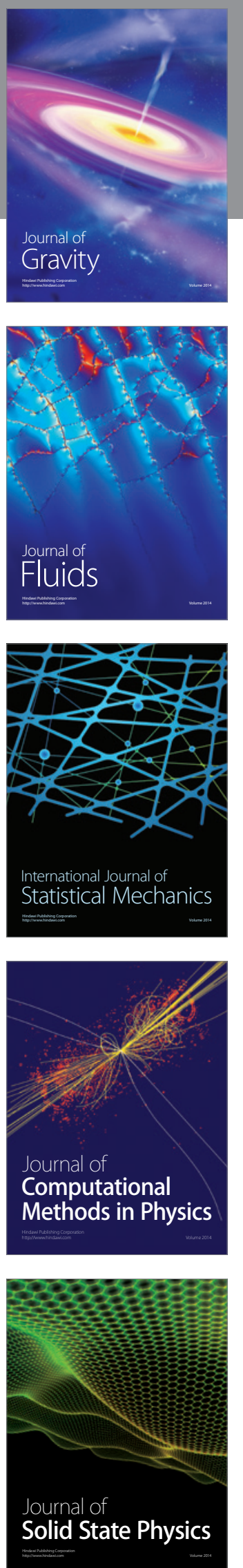
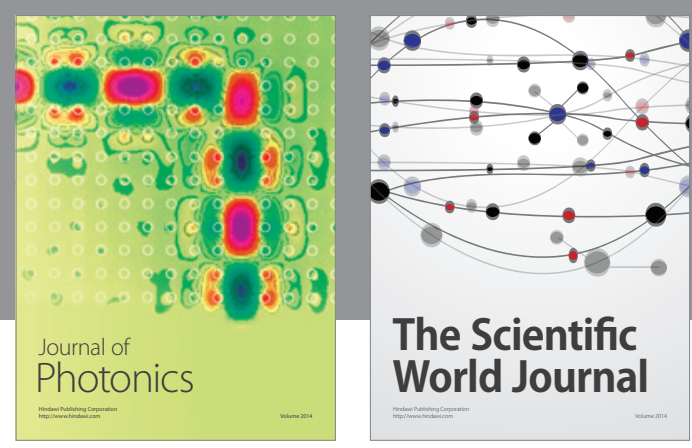

The Scientific World Journal

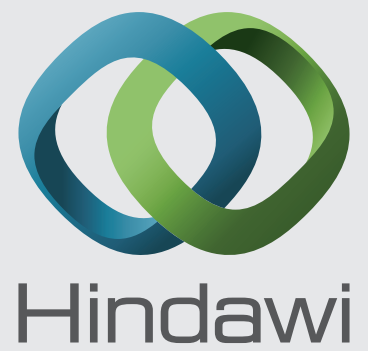

Submit your manuscripts at http://www.hindawi.com
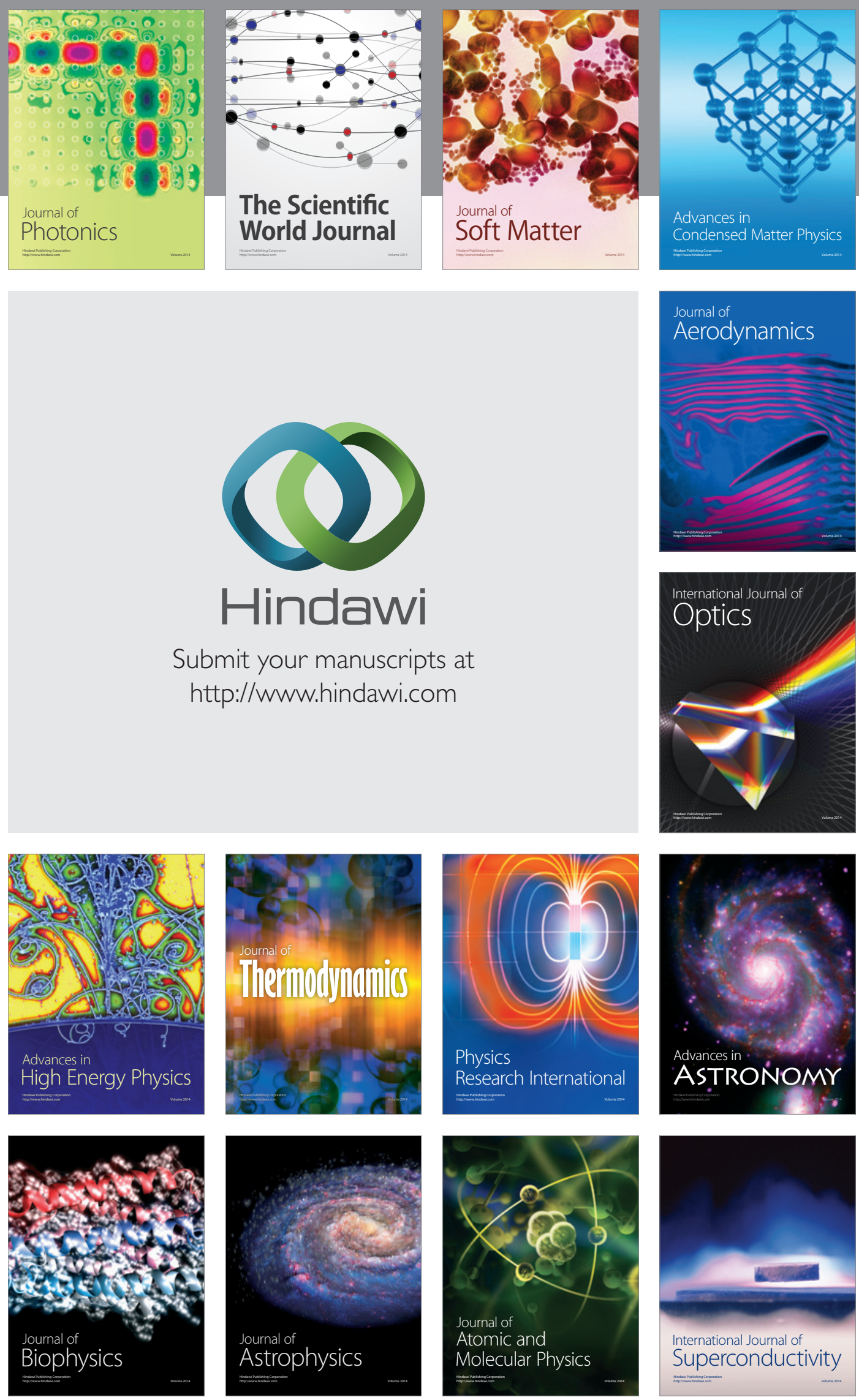
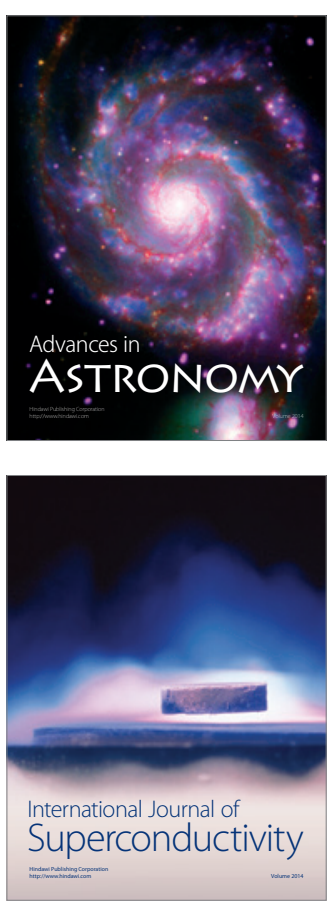